\title{
Performance Evaluation of OFDM System for Different Modulation Techniques on the basis of Bit Error Rate and Peak to Average Power Ratio
}

\author{
Gagandeep Kaur \\ Research Scholar, \\ Indus Institute of Engineering and Technology, Jind, \\ Haryana, India
}

\author{
Shelly Garg \\ Professor, \\ Electronics and Communication Engineering \\ Indus Institute of Engineering and Technology, Jind, \\ Haryana, India
}

\begin{abstract}
Orthogonal frequency division multiplexing (OFDM) is a form of digital modulation used in a wide array of communication systems. This paper presents a comprehensive performance analysis of orthogonal frequency division multiplexing system. We investigate the basic principle of OFDM system and through computer simulation we present the Bit error rate(BER) and peak-to-average power ratio(PAPR) of OFDM system for different modulation techniques.
\end{abstract}

\section{Keywords}

Bit error rate (BER), Peak-to-average power ratio, orthogonal frequency division multiplexing (OFDM)

\section{INTRODUCTION}

Wireless communication system is growing fastly in communication industry. The use of internet has been growing as number of users and amount of information contents are increasing day by day.3rd Generation system has been rolled in many countries as it has high data rate, as well as high spectral efficiency. LTE [2] establish as the latest step towards the 4 th generation $(4 \mathrm{G})$ of radio technologies. It is designed to increase the capacity and speed of mobile communications. LTE a cellular wireless communication standard based on orthogonal frequency-division multiplexing (OFDM). Orthogonal Frequency Division Multiplexing (OFDM) is a special form of multicarrier (MC)[3] that dates back to $1960 \mathrm{~s}$ [1]. The primary goal of next-generation wireless systems (4G) will not only be the introduction of new technologies to cover the need for higher data rates and new services, but also the integration of existing technologies in a common platform.

The next generation mobile technology with more advance features. Orthogonal Frequency Division Multiplexing (OFDM) is a Multi-carrier modulation technique in which available spectrum is divided into several narrow bands and data is divided into parallel data streams, each transmitted on a separate band. The concept of using parallel data transmission by means of OFDM was proposed by Chang in 1966 [4] for dispersive fading channels. In 1971, Weinstein and Ebert [5] proposed time-limited MC transmission, which is what we call OFDM today.

In this paper, we are introduce the basic principle of OFDM system. Futhermore through computer simulation, we first present the BER performance of OFDM system for different modulation techniques (QPSK, M-QAM where $\mathrm{M}$ is $8,16,32,64,128,256)$ and secondly PAPR characteristics using the complementary cumulative distribution function (CCDF).

\section{OFDM System}

OFDM is one of multicarrier transmission methods and perhaps, the most important between them. In OFDM input data is transmitted on one of available subcarriers.

OFDM system is similar to traditional FDM systems. But in OFDM system there is no need for wide guard band to provide reliability which was in FDM

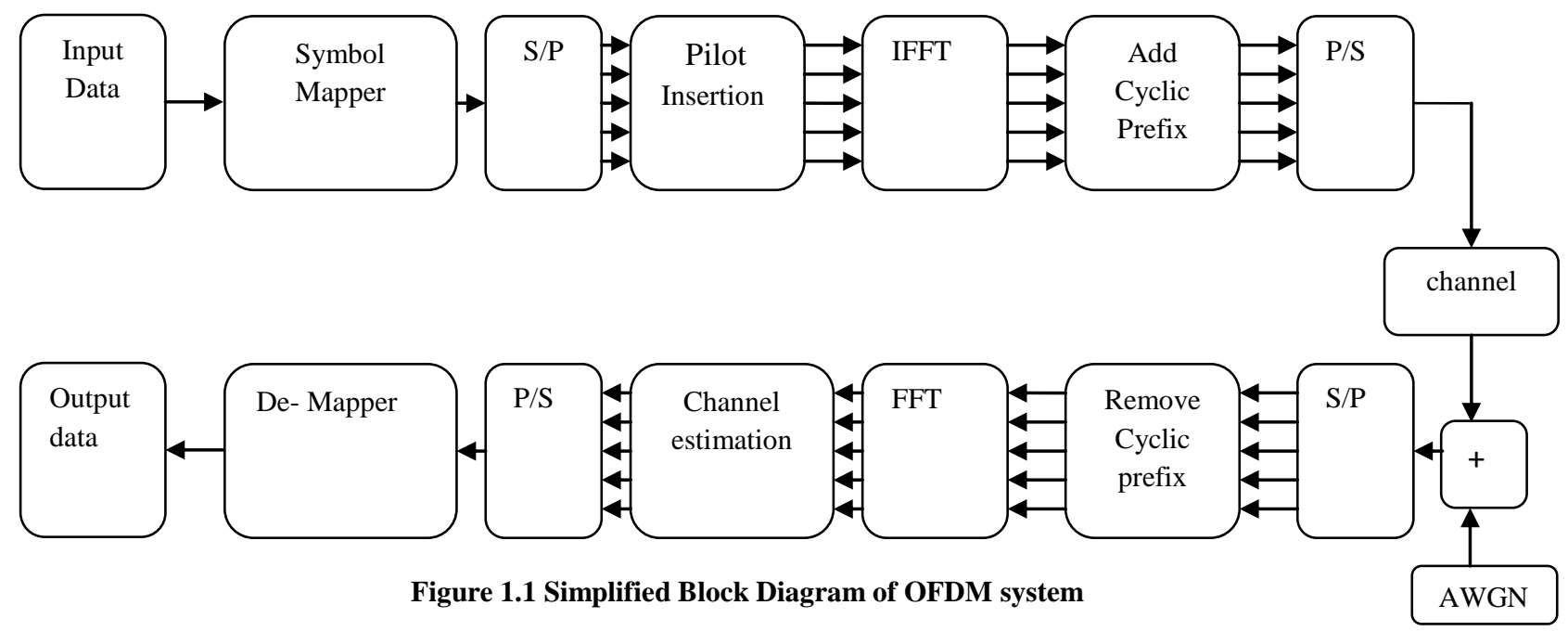


However, in order to be able to restore the transmitted symbols in the receiver, subcarriers should be located in frequency domain such that the energy of the interference caused from each subcarrier becomes exactly zero at the central frequency of the others. Therefore, the signal modulated on each subcarrier is 'orthogonal' to others and the data can be restored successfully at the receiver.

\section{Basic Principle of OFDM}

The system's basic principle is that the original bandwidth is divided into a high number of narrow sub-bands, in which the mobile channel can be considered non-dispersive [4]. Hence, no channel equalizer is needed. Figure1.1 Simplified block diagram of OFDM System. When data is transmitted from transmitter to receiver, during the transmission data bits suffers corruption due to presence of unwanted noise in the channel. Thus the error is obtained in the form of Bit Error Rate (BER). BER is one of the most important parameter of OFDM.

\section{SIMULATION RESULTS}

In OFDM System, PAPR and BER are two most important performance parameters. In this section, we are going to evaluate the PAPR and BER performances of OFDM System for different modulation techniques. For the purpose of comparing the performance of OFDM for QPSK, M-QAM where $M$ is $8,16,32,64,128,256$ we have developed the simulation model by using LabVIEW software. In our simulation we set the number of users $Q=4$, where all the users use the same transmit power. The number of subcarriers per user are $\mathrm{N}=32$, therefore the FFT size for OFDM is $\mathrm{M}=$ $\mathrm{N}=32$, Here we estimate BER with respect to different SNR for OFDM system performances of OFDM via computer simulations and verify our results with the results given in [10] [11]. The results obtained are shown in figures 1.3 To 1.9 respectively. In all the figures presenting BER performance, horizontal axes indicate the signal-to-noise ratio (SNR) in $\mathrm{dB}$ and vertical axes is the BER over 1000 iteration. It has been observed from the figures that for different modulation techniques as SNR increases, BER decreases. The different values of BER for different modulation technique at SNR $30 \mathrm{~dB}$ is compared and given in tabular form as follows:

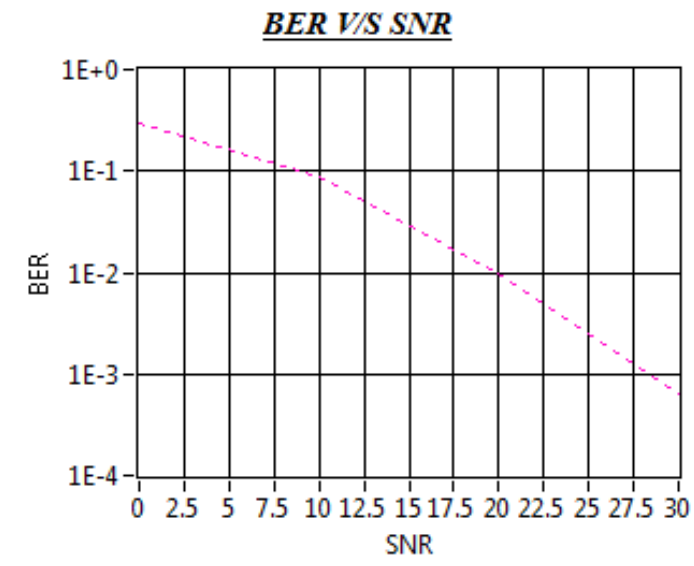

Figure 1.3 BER performance of OFDM system using QPSK for $\mathrm{M}=32$

Following Figure 1.10 shows the front panel of OFDM system model

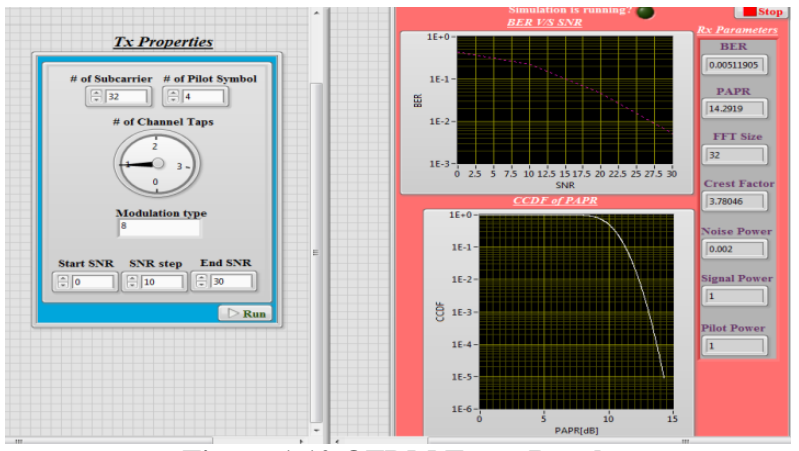

Figure 1.10 OFDM Front Panel

BER V/S SNR

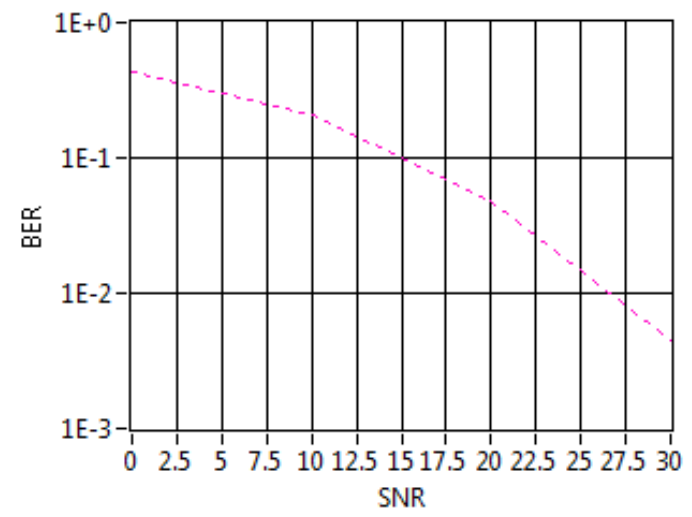

Figure 1.4 BER performance of OFDM system using 8QAM for $M=32$

BER V/S SNR

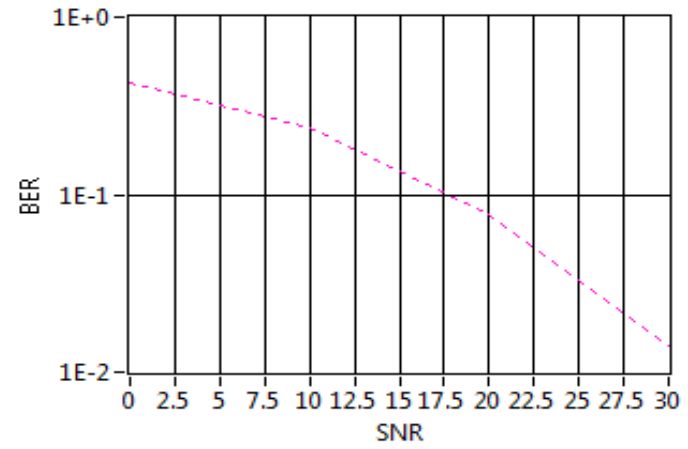

Figure 1.5 BER performance of OFDM system using 16QAM for $M=32$

\section{BER V/S SNR}

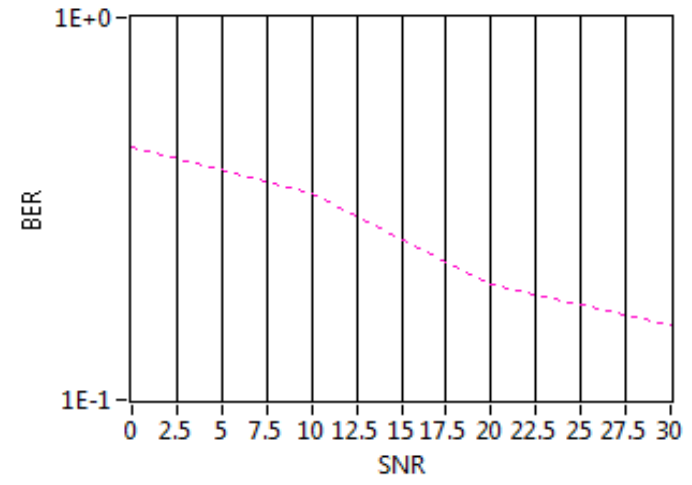


Figure 1.6 BER performance of OFDM system using 32QAM for $M=32$

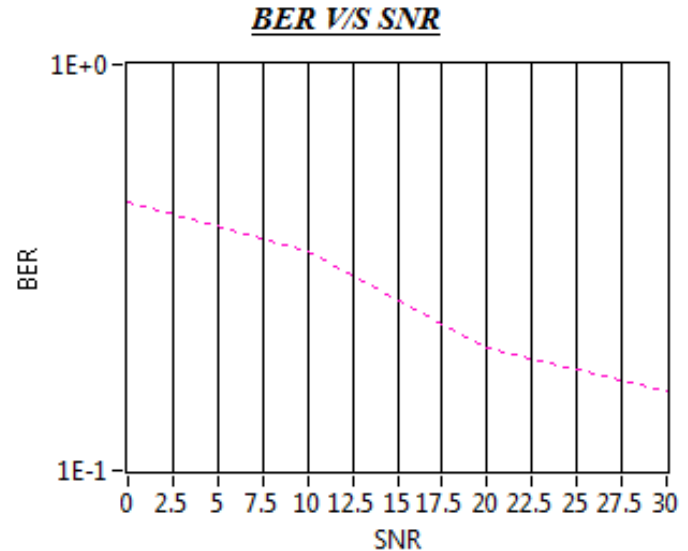

Figure 1.7 BER performance of OFDM system using 64QAM for $M=32$

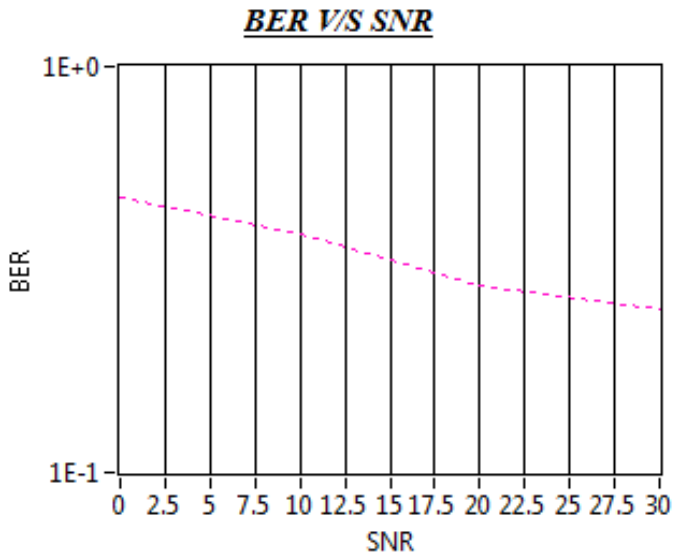

Figure 1.8 BER performance of OFDM system using 128QAM for $M=32$

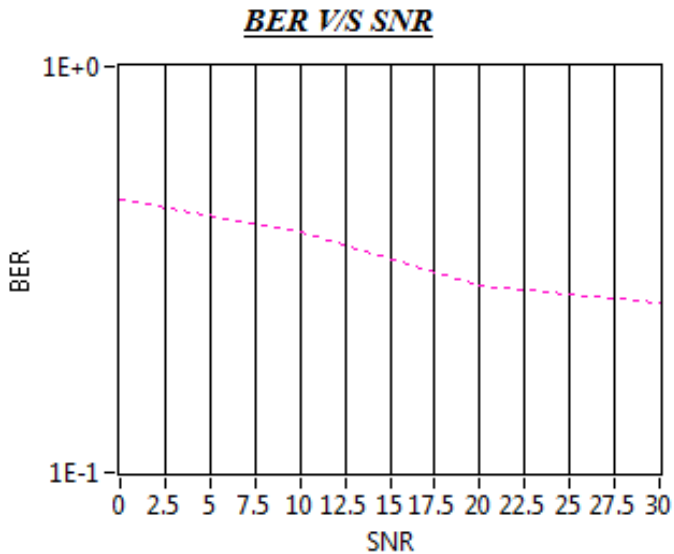

Figure 1.9 BER performance of OFDM system using 256QAM for $M=32$
Table 1.1 BER values of OFDM system for different modulation for $\mathrm{M}=32$

\begin{tabular}{|c|c|}
\hline $\begin{array}{c}\text { Modulation } \\
\text { Techniques }\end{array}$ & $\begin{array}{c}\text { BER Values at SNR } \\
\text { 30dB }\end{array}$ \\
\hline QPSK & 0.00169643 \\
\hline 8-QAM & 0.00511905 \\
\hline 16-QAM & 0.0144196 \\
\hline 32-QAM & 0.116929 \\
\hline 64-QAM & 0.158393 \\
\hline 128-QAM & 0.243163 \\
\hline 256-QAM & 0.256317 \\
\hline
\end{tabular}

After examining the simulation results, it is concluded that OFDM exhibits best BER performance for QPSK modulation. Further, 8-QAM and 16-QAM also provide good BER performance and rest of the BER results are shown in table 1.1 given above. These results shown above are for $\mathrm{M}=32$.

Where $\mathrm{M}=$ number of subcarriers. Similarly, BER performance for $\mathrm{M}=64$ and 128 are shown in the Tables 1.2 and 1.3 respectively. In both tables, Among all modulation techniques, we again find out that OFDM provides best BER results for QPSK modulation, followed by 8-QAM and 16QAM and average BER performance is obtained for rest of the modulation techniques.

Table 1.2 BER values of OFDM system for different modulation for $M=64$

\begin{tabular}{|c|c|}
\hline $\begin{array}{l}\text { Modulation } \\
\text { Techniques }\end{array}$ & $\begin{array}{c}\text { BER Values at SNR } \\
\text { 30dB }\end{array}$ \\
\hline QPSK & 0.00258929 \\
\hline 8-QAM & 0.00535714 \\
\hline 16-QAM & 0.0164063 \\
\hline 32-QAM & 0.109804 \\
\hline 64-QAM & 0.164851 \\
\hline 128-QAM & 0.245051 \\
\hline 256-QAM & 0.254018 \\
\hline
\end{tabular}

Table 1.4 BER values of OFDM system for different number of Subcarrier using QPSK 
Table 1.3 BER values of OFDM system for different modulation for $M=128$

\begin{tabular}{|c|c|}
\hline $\begin{array}{c}\text { Modulation } \\
\text { Techniques }\end{array}$ & $\begin{array}{c}\text { BER Values at SNR } \\
\text { 30dB }\end{array}$ \\
\hline QPSK & 0.00169643 \\
\hline 8-QAM & 0.00511905 \\
\hline 16-QAM & 0.0144196 \\
\hline 32-QAM & 0.116929 \\
\hline 64-QAM & 0.158393 \\
\hline 128-QAM & 0.243163 \\
\hline 256-QAM & 0.256317 \\
\hline
\end{tabular}

\section{PAPR Performance of OFDM system}

We have also run the simulation model for obtaining PAPR performance and verified our results with the results given in [12]. We run the simulation model for $\mathrm{M}=32,64$, and 128 , then we find out the PAPR individually. Figure 1.11, 1.12, and 1.13 shows comparison of CCDF of PAPR for the OFDM system. The PAPR values so obtained are also tabulated in Table 1.5

Table 1.5 PAPR (dB) values of OFDM system for different number of Subcarriers

\begin{tabular}{|cccc|}
\hline $\begin{array}{c}\text { No. of } \\
\text { Subcarriers }\end{array}$ & 32 & 64 & 128 \\
\hline PAPR (dB) & 12.5959 & 17.6772 & 18.9774 \\
\hline
\end{tabular}

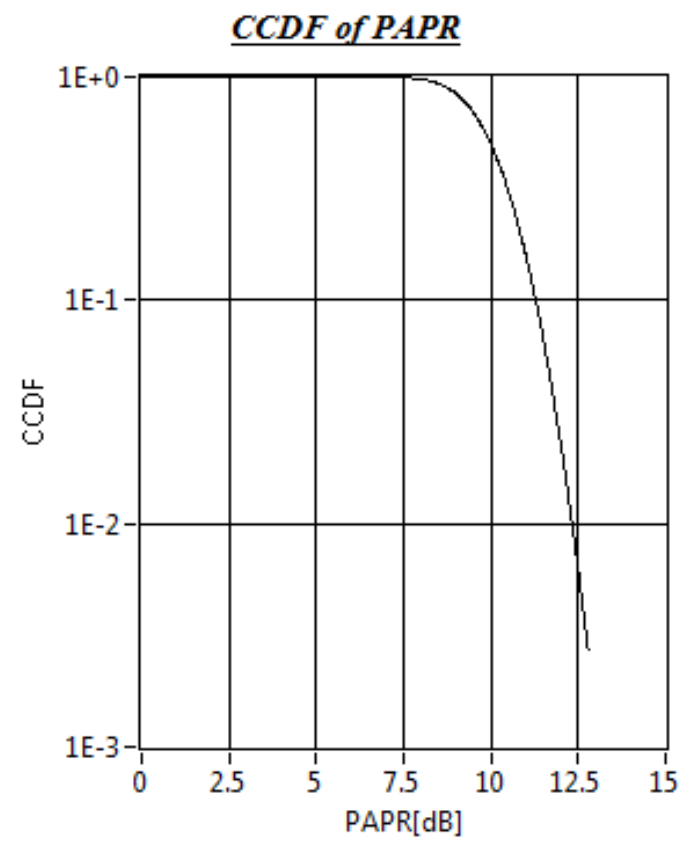

Figure 1.11 PAPR performance of OFDM system for $\mathbf{M}=32$

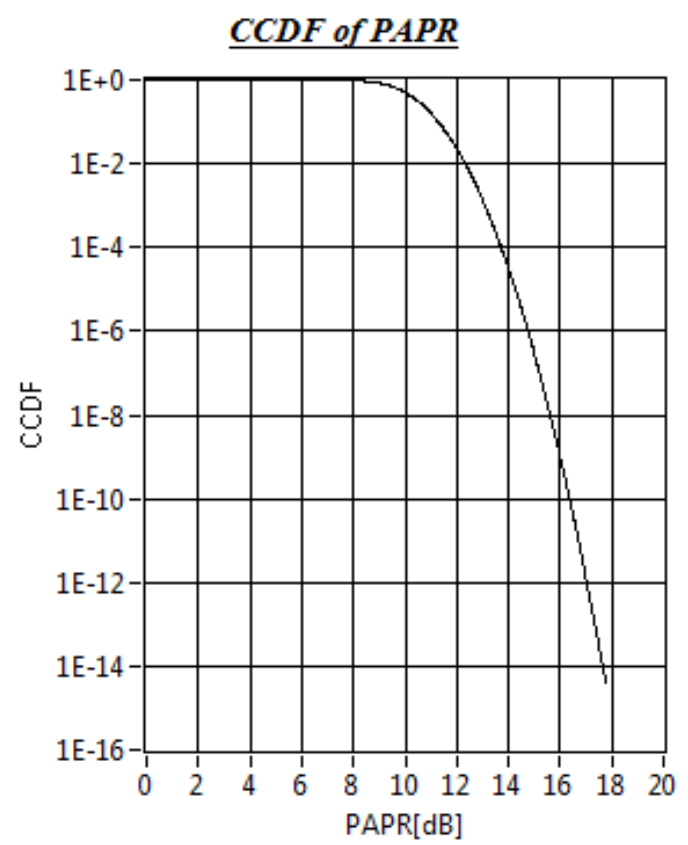

Figure 1.12 PAPR performance of OFDM system for $M=64$

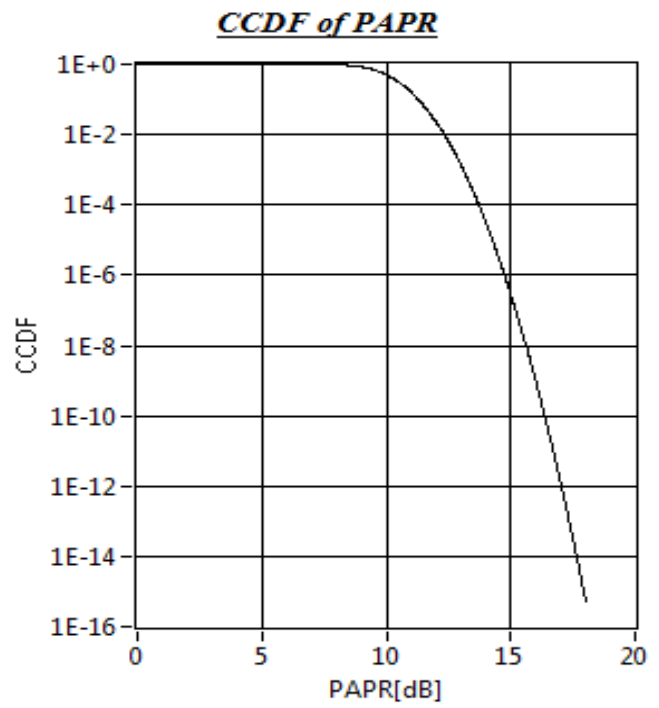

Figure 1.13 PAPR performance of OFDM system for $M=64$

Thus it is clearly noticed that the PAPR values for $M=32,64$, and 128 are quite high.

\section{RESULTS AND DISCUSSIONS}

After observing all the simulation results, it is concluded that OFDM exhibits best BER performance for QPSK modulation. Further, 8-QAM and 16-QAM also provide good BER performance. The PAPR values for $\mathrm{M}=32,64$, and 128 are quite high and this is one of the main drawbacks of OFDM system. This high value of PAPR can be controlled by proper subcarrier mapping in OFDM system.

\section{REFERENCES}

[1] T. Hwang, C. Yang et al. "OFDM and Its Wireless Applications: A Survey," IEEE Transaction on Vehicular Technology, Vol. 58, No. 4, May 2009, pp. 1663-1694. 
[2] Dongwoon Bai et al. "LTE-Advanced Modem Design: Challenges and Perspectives," IEEE Communication Magazine, Vol. 50, Issue 2 February 2012, pp. 178-186.

[3] Shinsuke Hara "Multicarrier Techniques For 4G Mobile Communications," Artech House Publisher, 2003

[4] L. Hanzo and T. Keller, "OFDM and MC-CDMA A Primer," John Wiley \& Sons Ltd, England, 2006.

[5] T. Hwang, C. Yang et al. "OFDM and Its Wireless Applications: A Survey," IEEE Transaction on Vehicular Technology, Vol. 58, No. 4, May 2009, pp. 1663-1694.

[6] K.Fazel "Multi-Carrier and Spread Spectrum Systems," John Wiley \& Sons Ltd, 2003.

[7] Ahmed R.S. Bahai, "Multi-Carrier Digital Communications," springer 2004.

[8] Shinsuke Hara "Multicarrier Techniques for 4G Mobile Communications," Artech House Publisher, 2003.
[9] Ahmed R.S. Bahai, "Multi-Carrier Digital Communications," springer 2004.

[10] F. Adachi, D. Garg et al. "Broadband Cdma Techniques," IEEE Journals on Wireless Communications, Vol. 12, Issue 2, April 2005, pp. 8-18.

[11] Aftab A. Memon, Syed M. Zafi et al. "Performance Comparison of OFDM, MC-CDMA and OFCDM for 4G Wireless Broadband Access and Beyond," Progress In Electromagnetics Research Symposium (PIERS) Proceedings, Marrakesh, Morocco, March, 2011 pp.1396-1399.

[12] Shinsuke Hara, Ramjee Prasad, "Design and Performance of Multicarrier CDMA System in Frequency-Selective Rayleigh Fading Channels," IEEE Transaction on Vehicular Technology, Vol. 48, No. 5, September 1999, pp. 1584-1595. 